\title{
A Full-Duplex Diversity-Assisted Hybrid Analogue/Digitized Radio Over Fibre for Optical/Wireless Integration
}

\author{
Varghese Antony Thomas, Salman Ghafoor, Mohammed El-Hajjar, and Lajos Hanzo
}

\begin{abstract}
A duplex Radio Over Fibre (ROF) ring architecture is proposed taking into account the constraints imposed by the cost of fibre laying and of the optical/electronic components, as well as the spectral efficiency and the duplex link performance. It has been shown that relying on Analogue ROF (AROF) and stateof-the-art Digitized ROF (DROF) architectures for downlink and uplink transmission, respectively, attains a high-integrity duplex performance. A sophisticated amalgam of Optical Carrier Suppression (OCS), Code Division Multiplexing (CDM), optical frequency multiplexing, Optical Carrier Reuse (OCR) and distributed antennas is conceived.
\end{abstract}

Index Terms-Radio over fibre (ROF), optical carrier reuse (OCR), code division multiplexing (CDM), distributed antenna system (DAS).

\section{INTRODUCTION}

$\mathbf{T}$ HE provision of a high speed ubiquitous wireless coverage is essential for a vibrant economy. The objective is to have a long wireless device battery recharge period, which in turn necessitates small cells illuminated by Radio Access Points (RAPs), while minimizing the wireless path loss. Radio Over Fibre (ROF) technology meets these objectives by bringing the high-bandwidth and low-attenuation optical fibre link closer to the wireless user in a typical campus network. The RAPs are connected by fibre to a central Base Station (BS) and the resultant Distributed Antenna Systems (DASs) are capable of improving the coverage by achieving diversity gain [1]. Low-complexity RAPs [2] and low optical fibre installation cost are essential in making ROF systems cost-effective. Both Analogue ROF (AROF) [2] and Digitized ROF (DROF) [3] implementations are feasible. However, the optical link's nonlinearity and fibre-dispersion limit both the affordable fibrelength and the attainable performance of the low complexity AROF architecture [3]. In contrast to AROF, the baseband DROF relies on expensive Analogue to Digital Converters (ADCs) and Digital to Analogue Converters (DACs), but as a benefit, it is capable of operating over longer distances [3]. Consequently DROF may achieve the same BER as AROF at a lower optical transmit power. As a further advantage of DROF solutions, multiple users may be optically Code Division Multiplexed (CDM). The authors of [4] - [9] relied on Optical Carrier Reuse (OCR), which reduced the number

Manuscript received May 4, 2012. The associate editor coordinating the review of this letter and approving it for publication was T. Tsiftsis.

V. A. Thomas, M. El-Hajjar, and L. Hanzo are with the School of ECS, University of Southampton, SO17 1BJ, United Kingdom (e-mail: \{vat1g10, meh, lh\}@ecs.soton.ac.uk).

S. Ghafoor is with the National University of Sciences and Technology, Pakistan (e-mail: salman.ghafoor@seecs.edu.pk).

The financial support of the EU's Concerto Project, of the National University of Science and Technology (NUST), Pakistan, as well as that of the EPSRC, UK under the auspices of the India-UK Advanced Technology Centre and the China-UK Science Bridge is gratefully acknowledged.

Digital Object Identifier 10.1109/LCOMM.2012.122012.120975 of optical transmitters required at the BS, thereby leading to a cost reduction. However, the architectures of [4]-[6] required optical carrier recovery at the RAP by using expensive, high selectivity optical filtering. As a further improvement, in [7] duplex transmission has been implemented using two different optical carriers, which were generated from a single original wavelength using Optical Carrier Suppression (OCS). The authors of [8], [9] employed the concept of multiplexing baseband wired data and RF wireless signal by reusing the DL optical carrier for transmitting wired UL data. The Mobile Station's (MS's) UL signals arrive at the RAPs asynchronously owing to their different clock and carrier frequencies as well as distances. Furthermore, owing to imperfect power control and to the ubiquitous Multi User Interference (MUI) of the 3G-style Code Division Multiple Access (CDMA) wireless scheme, typically a lower SNR is experienced in the asynchronous wireless UL than in the synchronous DL [10]. Hence we design a more robust, but more costly DROF link for the UL optical transmission, while a low-cost AROF link is conceived for the DL in the architecture proposed in this letter. As a further benefit, the UL baseband DROF signal may be transmitted by modulating the same optical carrier used to transmit the DL AROF signal, facilitating OCR and hence a better optical spectral efficiency. The DL optical carrier may be reused due to the lower optical transmit power requirement of the DROF mode. The generation of the optical carriers for all the RAPs relies on a single central laser source used at the BS for exploiting the Mach Zehnder Modulator's (MZM) non-linearity [11]. This minimizes the cost of optical components. Finally, the use of ring topology is advocated, which minimises the cost of fibre laying. Thus, the campus network relies on a hybrid AROF and DROF architecture, bearing in mind the constraints imposed by the cost of both fibre-laying and of the optical/electronic components, as well as the objective of achieving a high optical spectral efficiency and a high UL/DL performance. The novel features of the proposed architecture are as follows:

1) Achieving full-duplex diversity assisted transmission by using AROF for the DL and DROF for the UL and hence improving the optical bandwidth efficiency with the aid of optical frequency multiplexing.

2) We dispense with optical carrier recovery for OCR and conceive a ring architecture relying on a single laser source at the BS to support both the UL and DL of multiple RAPs.

3) Using optical CDMA aided DROF for supporting multiple UL users.

We emphasize that DROF was preferred over Baseband ROF (BROF) as a benefit of its lower power consumption [12] and reduced-complexity RAPs [13]. Our paper is organized 


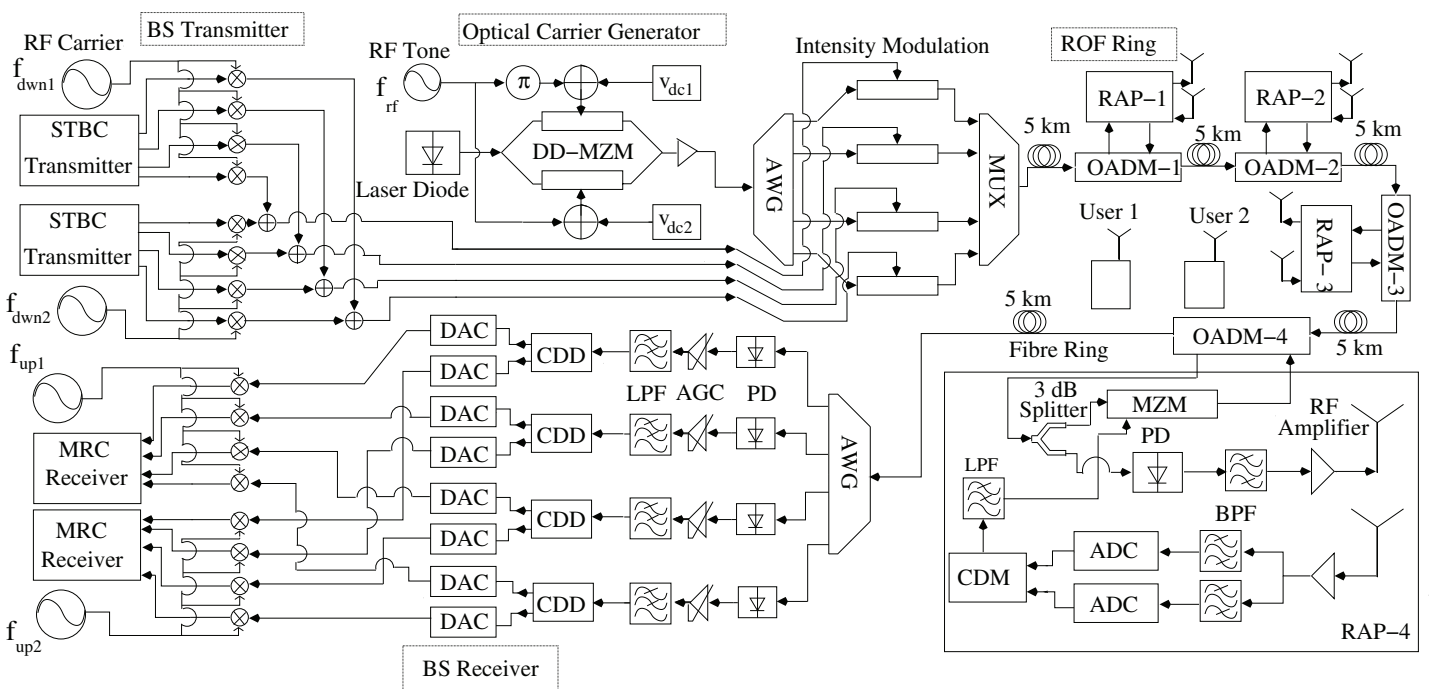

Fig. 1: Proposed system architecture.

TABLE I: Parameter values used in the ROF architecture

\begin{tabular}{|l|l|l|l|}
\hline Parameter & Value & Parameter & Value \\
\hline $\begin{array}{l}\text { RF DL } f_{d w n 1} \\
\& f_{d w n} 2\end{array}$ & $\begin{array}{l}2.47 \mathrm{GHz} \\
5.8 \mathrm{GHz}\end{array}$ & $\begin{array}{l}\text { PD receiver } \\
\text { Noise Figure }\end{array}$ & $6 \mathrm{~dB}$ \\
\hline $\begin{array}{l}\text { RF Modulation } \\
\text { scheme }\end{array}$ & $\begin{array}{l}\text { Binary Phase } \\
\text { Shift Keying }\end{array}$ & $\begin{array}{l}\text { PD receiver } \\
\text { Trans-impedance }\end{array}$ & $125 \Omega$ \\
\hline Fibre Dispersion & $16 \mathrm{ps} / \mathrm{km}-\mathrm{nm}$ & ADC Resolution & $4 \mathrm{bits}$ \\
\hline$\lambda_{\text {laser }}$ & $1550 \mathrm{~nm}$ & PD responsivity & $0.8 \mathrm{~A} / \mathrm{W}$ \\
\hline RF DL and & $32 \mathrm{MBPS}$ & RF UL $f_{u p 1}$ & $2.416 \mathrm{GHz}$ \\
UL Bit rate & $16 \mathrm{MBPS}$ & $\& f_{\text {up } 2}$ & $5.744 \mathrm{GHz}$ \\
\hline RF channel width & $60 \mathrm{MHz}(\mathrm{DL})$ & Bandpass & $64 \times 10^{6}$ \\
with guard band & $30 \mathrm{MHz}(\mathrm{UL})$ & sampling rate $f_{s}$ & sample/s \\
\hline RRC filter roll-off & 0.5 & $f_{r f}$ used in OCG & $12.5 \mathrm{GHz}$ \\
\hline DL RF & Space Time & length of & $25 \mathrm{~km}$ \\
Diversity Scheme & Block Coding & fibre ring & \\
\hline UL RF & Maximal Ratio & DD-MZM $t_{\text {attn }} \&$ & $4 \mathrm{~dB}$ \\
Diversity Scheme & Combining & Extinction Ratio $\epsilon$ & $35 \mathrm{~dB}$ \\
\hline
\end{tabular}

as follows. In section II and III we discuss the proposed architecture amd simulation results respectively, followed by the conclusions in section IV.

\section{Proposed ROP Network Architecture}

The complete ring architecture is shown in Fig. 1. The parameters used in the architecture are summarized in Table.1.

\section{A. Optical Carrier Generator (OCG)}

The optical carriers are generated at the BS by exploiting the Dual Drive (DD) MZM non-linearity with the aid of OCS [11]. The output of the Laser Diode (LD) operating at the optical transmit of power $P_{o p}$ and frequency of $f_{\text {laser }}$ (wavelength $\lambda_{\text {laser }}$ ) is:

$$
E_{\text {laser }}(t)=\left(\sqrt{2 P_{o p}}\right) e^{j 2 \pi f_{\text {laser }}} .
$$

The drive voltages applied to the DD-MZM arms are $v_{1}(t)=v_{d c 1}+v_{r f} \cos \left(2 \pi f_{r f} t+\theta_{1}\right)$ and $v_{2}(t)=v_{d c 2}+$ $v_{r f} \cos \left(2 \pi f_{r f} t+\theta_{2}\right)$, where $v_{d c}, \quad v_{r f}$ and $\theta$ are the DC bias, RF tone amplitude and phase, respectively. The output

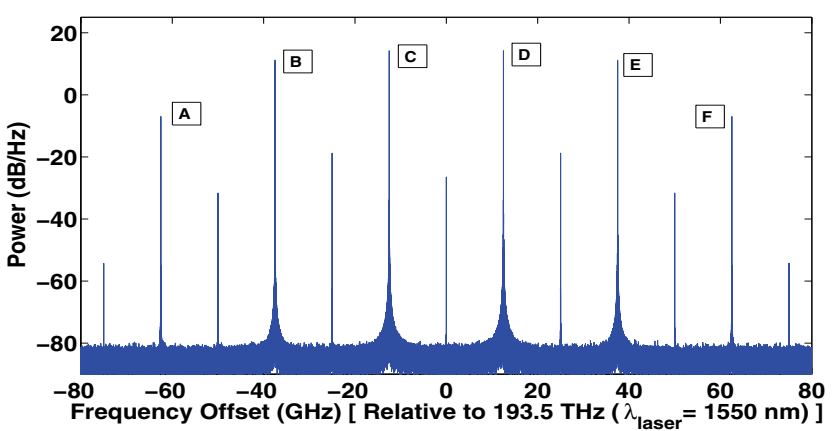

Fig. 2: The OCG's output spectrum for $35 \mathrm{~dB}$ extinction ratio.

optical field of the DD-MZM having an insertion loss of $t_{\text {att }}$ and extinction ratio $\epsilon$ is:

$$
E_{M Z M-O C G}=\frac{E_{\text {laser }}(t) \sqrt{t_{\text {attn }}}}{2}\left[e^{j \pi \frac{v_{1}(t)}{v_{\pi}}}+\gamma e^{j \pi \frac{v_{2}(t)}{v_{\pi}}}\right] .
$$

Here $v_{\pi}$ is the drive voltage that results in $180^{\circ}$ phase shift in each arm and the parameter $\gamma$ accounts for the non-ideal behaviour of the DD-MZM, where $\gamma=(\sqrt{\epsilon}-1) /(\sqrt{\epsilon}+1) \approx 1$. A high value of $v_{r f}=0.9 v_{\pi}$ was chosen to generate strong harmonics. For $\epsilon=35 d B$ (i.e. $\gamma=0.965), v_{d c 1}=v_{\pi}, v_{d c 2}=$ $0, \theta_{1}=\pi, \theta_{2}=0$ and $t_{\text {attn }}=4 d B$ Eq. (2) may be rewritten using Bessel functions as [11]:

$$
\frac{0.63 E_{\text {laser }}(t)}{2}\left(\sum_{n=-\infty}^{\infty} j J_{n Z M-O C G}(2.82)\left(e^{j n \pi}-\gamma\right) e^{j n\left(2 \pi f_{r f} t+\pi\right)}\right),
$$

where $J_{n}$ is the order $n$ Bessel function of the first kind. As seen from Fig. 2, the output spectrum has six significant side bands (A-F) spaced at $2 f_{r f} \mathrm{~Hz}$ that may be used as optical carriers in a Wavelength Division Multiplexing (WDM) application by employing an Arrayed Waveguide Grating (AWG) that selects these significant sidebands and has a channel spacing of $2 f_{r f} \mathrm{~Hz}$. Moreover an Optical Add Drop Multiplexer (OADM) can be used to select a single significant sideband. 


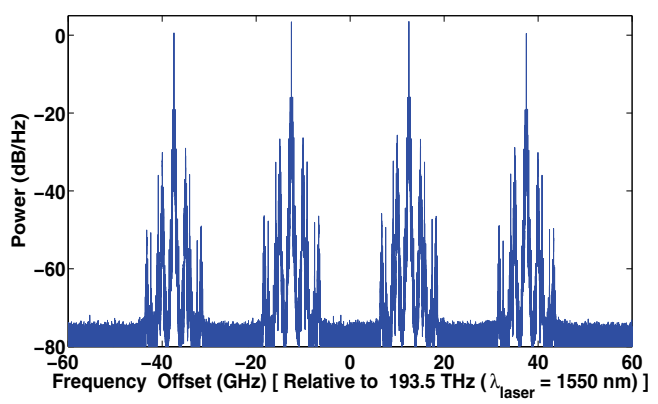

(a) Optical spectrum at the input of the BS receiver.

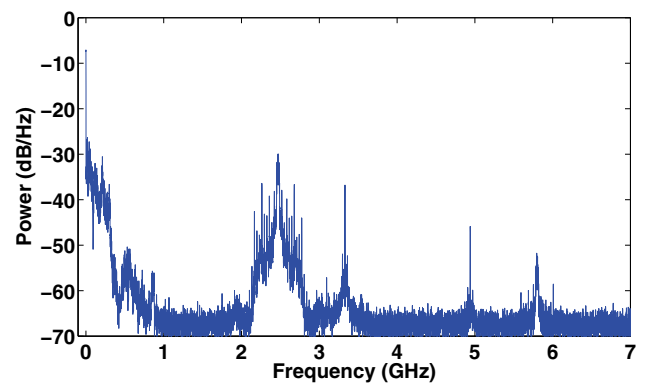

(b) Electronic spectrum after photodetection of the RAP UL signal.

Fig. 3: Optical and electronic spectrums after propogation along the ROF ring.

\section{B. BS Transmitter}

The DL RF signals transmitted to each RAP at frequencies $f_{d w n 1}=2.47 \mathrm{GHz}$ and $f_{d w n 2}=5.8 \mathrm{GHz}$ are Sub-Carrier Multiplexed (SCM) and used for intensity modulating the optical sidebands B to E at the output of OCG. Each of these sidebands now transmits a Double Side-Band (DSB) DL optical signal to the 4 RAPs. The intensity $x_{i}(t)$ of the DL optical signal transmitted to RAP-i is:

$$
x_{i}(t)=1+k_{1}\left[m_{1, i}(t)+m_{2, i}(t)\right]+h(t),
$$

where $m_{1, i}(t), m_{2, i}(t)$ are the DL RF signals of users 1 and 2 sent to RAP-i. Still referring to Eq. (4), $h(t)$ represents the weaker harmonics and $k_{1}$ depends on the optical modulation index. Transmit diversity was achieved with the aid of Space Time Block Coding (STBC) [10] following the generation of the Root Raised Cosine (RRC) filtered BPSK signals. A single user per RF carrier was assumed. However, the concept may be extended to support multiple users per RF carrier by using wireless BPSK-CDMA signals.

\section{ROF Ring}

The DL ROF signals are transmitted through a Single Mode Fibre (SMF) using the Split Step Fourier Method. We use the 4 major sidebands in the OCG output for supporting 4 RAPs in the ring. Duplex optical transmission was used by each RAP at a single OCG output sideband via an OADM and OCR. The block diagram of a RAP is shown in Fig. 1. The optical signal dropped at RAP-i is fed to a $3 \mathrm{~dB}$ splitter. One of the splitter outputs is photo-detected and amplified for recovering the DL RF signal, while the other output $\left(\tilde{x}_{i}(t)\right)$ is modulated by the UL baseband DROF signal $\left(b_{i}(t)\right)$. The baseband data modulates the reused DL optical carrier and corrupts the two DL sidebands, albeit fortunately this is not a problem, since the DL signal has already been detected. Using Eq. (4), the intensity of the UL optical signal transmitted from RAP-i, is:

$$
\begin{aligned}
y_{i}(t) & =\tilde{x}_{i}(t)\left[1+k_{2} b_{i}(t)\right] \\
& =1+k_{2} b_{i}(t)+k_{1} m_{1, i}(t)+k_{1} m_{2, i}(t) \\
& +k_{1} k_{2} m_{1, i}(t) b_{i}(t)+k_{1} k_{2} m_{2, i}(t) b_{i}(t) \\
& +k_{2} b_{i}(t) h(t)+h(t),
\end{aligned}
$$

where $k_{2}$ depends on the depth of the UL modulation. Since $\tilde{x}_{i}(t)$ is an attenuated and dispersion contaminated version of $x_{i}(t)$, they occupy the same frequency band. This permits the use of Eq. (4) in Eq. (5) for the above proof-of-concept mathematical analysis because our primary concern is the occupied frequency bands after UL optical modulation. Only the second term in Eq. (5) is a baseband term and the rest may readily be filtered out using an inexpensive electronic filter after intensity detection using a photodiode (PD). The users' wireless UL signals were received at all RAPs and then combined by a Maximal Ratio Combining (MRC) receive antenna diversity scheme [10] at the BS. In the UL optical transmitter at the RAP, using Nyquist sampling of the received BPSK RRC filtered RF signals at $f_{u p 1}$ and $f_{u p 2} \mathrm{~Hz}$ is not practically feasible. Hence digitization of these signals was performed using the concept of bandpass sampling. The valid bandpass sampling rates for a bandwidth of $\mathrm{B}$ are [3]:

$$
\frac{2\left(f_{u p}+\frac{B}{2}\right)}{k} \leq f_{s} \leq \frac{2\left(f_{u p}-\frac{B}{2}\right)}{k-1} \forall 1 \leq k \leq\left\lfloor\frac{f_{u p}+\frac{B}{2}}{B}\right\rfloor,
$$

where $\lfloor w\rfloor$ represents the integer part $w$ and $k \in I$. The use of BPSK modulation results in a low ADC/DAC resolution requirement. Nevertheless, the ADC resolution should guarantee that the link SNR is not dominated by the quantization noise [3]. Using the equations in [3], a resolution of 4 bits/sample was found to satisfy this criterion, when using inexpensive ADCs having an rms jitter of approximately 10ps. The pair of digitized RF signals were then code division multiplexed using orthogonal Walsh codes [10] prior to optical modulation and transmission over the synchronous optical UL.

\section{BS Receiver}

The spectrum of the UL ROF signal input to the BS receiver is shown in Fig. 3(a). As seen from Eq. (5), each of the sidebands B, C, D and E carries the CDM DROF baseband UL signal and the corrupted DL RF sidebands. The UL optical signal is fed into a $1 \times 4$ AWG, each of whose outputs are photo-detected and amplified using an Automatic Gain Control (AGC) amplifier. The amplified signals are Low Pass Filtered (LPF) to remove the corrupted DL RF signals and their intermodulation products. Fig. 3(b) shows the spectrum of the photodetected signal prior to filtering. The filtered baseband CDM DROF signals are code division de-multiplexed (CDD) to separate the two digitized UL RF signals received from each RAP, which are then fed into DACs for recovering the RF signals. Since the UL wireless signals arriving from the two mobile users are received at all the RAPs, four copies of 


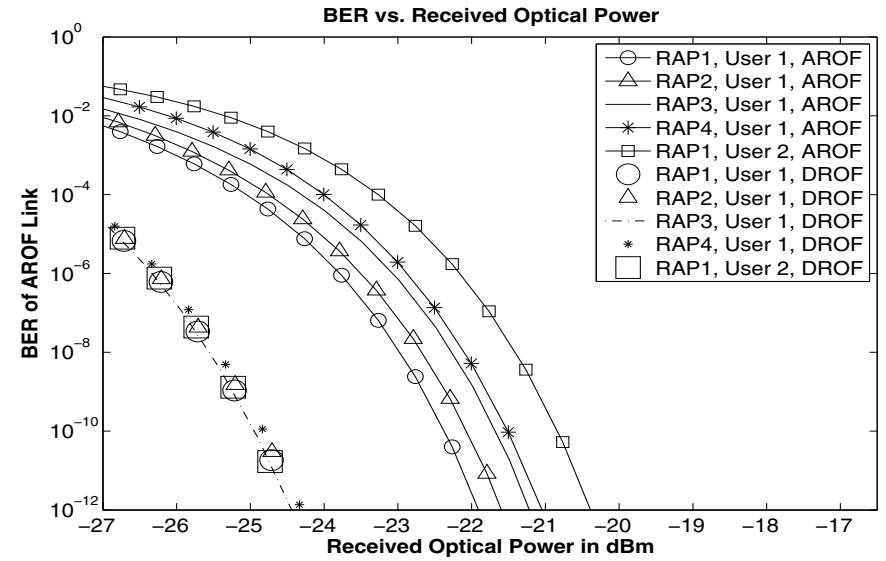

(a) BER performance of the AROF and DROF links.

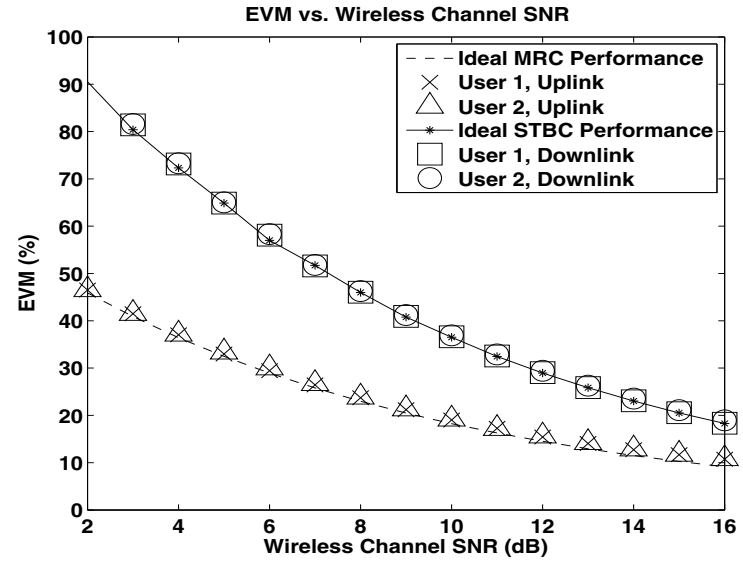

(b) Uplink performance using MRC \& BPSK modulation and downlink performance using STBC \& BPSK modulation.

Fig. 4: System performance results.

the two UL RF signals are obtained. These are then downconverted and fed to MRC detection blocks [10].

\section{RESULTS}

It can be observed from Fig. 4a that the sensitivity of the AROF link varies more significantly with the changes in the user's RF carrier as well as with the DL fiber length as compared to the DROF link, which is due to the increased effects of chromatic dispersion and owing to the link's nonlinearity. Moreover, the performance of the DROF link was found to be robust, despite reusing the DL optical carrier and the MZM non-linearity. It is envisaged that the ROF technology will be extensively used in future RF systems, especially at mm-wave frequencies. Having a reduced AROF sensitivity, inflicted by the higher levels of chromatic dispersion, would further increase the level of signal corruption imposed by the optical link at higher RF carriers. This makes DROF more attractive for transporting the lower-integrity UL signal, when relying on OCR. Fig. 4b characterizes the overall system performance, when both UL and DL diversity was exploited and $15 \mathrm{dBm}$ of optical power entered the BS transmitter AWG. The wireless channel symbol energy was $E_{s}$ and noise spectral density was $N_{o}$. Using STBC transmit-diversity in the DL and MRC receive-diversity in the UL helped combat the wireless channel's Rayleigh fading and hence improved the coverage quality. We emphasize here that despite the strong dependence of the AROF link's sensitivity on the RF carrier, the overall DL system performance does not vary much between the users, since the lower-SNR wireless channel dominates the overall system performance. This however would not be the case for higher RF carriers.

\section{CONCLUSIONS}

In this letter a wireless campus network architecture has been proposed that effeciently utilises the optical bandwidth and minimizes the implementation cost, while maintaining robust duplex performance. It uses AROF for the DL, while having a lower transmit power requirement enables the use of OCR aided DROF for the UL. We dispense with the need for optical carrier recovery for OCR and conceive an architecture that utilises a single laser source at the BS to support both the UL and DL of multiple RAPs. The ability of DROF to support multiple UL users through CDM has also been shown.

\section{REFERENCES}

[1] P. Monteiro, S. Pato, Lo, E. Pez, D. Wake, N. J. Gomes, and A. Gameiro, "Fiber optic networks for distributed radio architectures: FUTON concept and operation," in Proc. 2010 IEEE Wireless Communications and Networking Conference Workshops, pp. 1-5.

[2] N. Mohamed, S. M. Idrus, and A. B. Mohammad, "Review on system architectures for the millimeter-wave generation techniques for RoF communication link," in Proc. 2008 IEEE International RF and Microwave Conference, pp. 326-330.

[3] P. A. Gamage, A. Nirmalathas, C. Lim, D. Novak, and R. Waterhouse, "Design and analysis of digitized RF-over-fiber links," J. Lightwave Technol., vol. 27, no. 12, pp. 2052-2061, June 2009.

[4] M. Bakaul, A. Nirmalathas, C. Lim, D. Novak, and R. B. Waterhouse, "Investigating the performance of star-tree and ring-bus fibre-radio networks incorporated with cascaded WDM optical interfaces," in Proc. 2008 International Topical Meeting on Microwave Photonics, pp. 225228

[5] A. Kaszubowska, L. Hu, and L. Barry, "Remote downconversion with wavelength reuse for the radio/fiber uplink connection," IEEE Photonics Technol. Lett., vol. 18, no. 4, pp. 562-564, Feb. 2006.

[6] L. Chen, H. Wen, and S. Wen, "A radio-over-fiber system with a novel scheme for millimeter-wave generation and wavelength reuse for up-link connection," IEEE Photonics Technol. Lett., vol. 18, no. 19, pp. 20562058, Oct. 2006.

[7] L. Chen, Y. Shao, X. Lei, H. Wen, and S. Wen, "A novel radio-overfiber system with wavelength reuse for upstream data connection," IEEE Photonics Technol. Lett., vol. 19, no. 6, pp. 387-389, Mar. 2007.

[8] S. Pan and J. Yao, "Simultaneous provision of UWB and wired services in a WDM-PON network using a centralized light source," IEEE Photonics J., vol. 2, no. 5, pp. 712-718, Oct. 2010.

[9] H. Chien, A. Chowdhury, Y. Hsueh, Z. Jia, S. Fan, J. Yu, and G. K. Chang, "A novel $60-\mathrm{GHz}$ millimeter-wave over fiber with independent 10-Gbps wired and wireless services on a single wavelength using PolMUX and wavelength-reuse techniques," in Proc. 2009 Conference on Optical Fiber Communication, pp. 1-3.

[10] L. Hanzo, L. L. Yang, E. L. Kuan, and K. Yen, Single and Multicarrier DS-CDMA: Multi User Detection, Space-Time Spreading, Synchronization, Standards and Networking, 1st edition. John Wiley \& Sons, 2003.

[11] S. Ghafoor and L. Hanzo, "Reduced dispersion duplex DQPSK radioover-fiber communications using single-laser-based multiple side-bands," in Proc. 2011 IEEE International Conference on Communications, pp. $1-5$.

[12] Y. Yang, C. Lim, and A. Nirmalathas, "Comparison of energy consumption of integrated optical-wireless access networks," in Proc. 2011 Optical Fiber Communication Conference and Exposition, pp. 1-3.

[13] C. Lim, A. Nirmalathas, Y. Yang, D. Novak, and R. B. Waterhouse, "Radio-over-fiber systems," in Proc. 2009 Communications and Photonics Conference and Exhibition, pp. 1-10. 